\title{
Microbiological characterization of Streptococcus pneumoniae and non-typeable Haemophilus influenzae isolates as primary causes of acute otitis media in Bulgarian children before the introduction of conjugate vaccines
}

Lena P Setchanova ${ }^{1 *}$, Tomislav Kostyanev ${ }^{1}$, Alexandra B Alexandrova', Ivan G Mitov' ${ }^{1}$ Dimitar Nashev² and Todor Kantardjiev²

\begin{abstract}
Background: Pneumococcal and Haemophilus influenzae type b (Hib) vaccines were introduced in our national immunisation program in April 2010. The aims of this retrospective, laboratory-based study were to determine the serotypes and antibiotic resistance of Streptococcus pneumoniae and H. influenzae isolates from middle ear fluid (MEF) collected before the introduction of immunization.

Methods: S. pneumoniae $(n=128)$ and $H$. influenzae $(n=40)$ strains isolated from MEF of children with AOM between 1994 and 2011 were studied. MICs were determined by a microdilution assay. Serotyping of S. pneumoniae was done by Quellung method and PCR capsular typing was used for H. influenzae. Macrolide resistance genes were detected by PCR for erythromycin resistant S. pneumoniae (ERSP). DNA sequencing of ftsl gene was performed for ampicillin nonsusceptible H. influenzae.

Results: The most common serotypes found among children with pneumococcal AOM were $19 \mathrm{~F}(20.3 \%), 6 \mathrm{~B}$ (15.6\%), and 19A (10.9\%). The potential coverage rates by the PCV7, PCV10 and PCV13 of children aged $<5$ years were $63.6 \%, 66.4 \%$ and $85.5 \%$, respectively. Reduced susceptibility to oral penicillin was seen in $68.1 \%$; resistance to erythromycin was $46.9 \%$. We found erm(B) gene in $56.7 \%$ of the ERSP, mef(E) gene in 25\%; $15 \%$ harbored both genes erm $(B)+\operatorname{mef}(E)$ and $3.3 \%$ had mutations of $L 4$ ribosomal protein. Of the $40 \mathrm{H}$. influenzae isolates $97.5 \%$ were nontypeable. Nonsusceptibility to ampicillin occurred in 25\%. Ampicillin resistance groups were: $\beta$-lactamase -positive ampicillin resistant (BLPAR) strains (10\%), $\beta$-lactamase-negative ampicillin resistant (BLNAR) strains (12.5\%) and $\beta$-lactamase-positive amoxicillin-clavulanate resistant (BLPACR) strains (2.5\%). Among BLNAR and BLPACR most of the isolates (5/6) belonged to group II, defined by the Asn526Lys substitution.
\end{abstract}

Conclusions: The levels of antibiotic resistance among S. pneumoniae and H. influenzae causing severe AOM in children are high in our settings. The existence of multidrug-resistant S. pneumoniae serotype 19A is of particular concern. The rate of BLNAR and BLPACR strains among $\mathrm{H}$. influenzae isolates was $15 \%$.

Keywords: AOM, S. pneumoniae, H. influenzae, Serotypes, Antibiotic resistance

\footnotetext{
* Correspondence: lenasetchanova@hotmail.com

'Department of Medical Microbiology, Medical University of Sofia, Faculty of Medicine, Sofia 1431, BULGARIA

Full list of author information is available at the end of the article
} 


\section{Background}

Acute otitis media (AOM) is the most common infection in children and the most common reason for which antibiotics are prescribed in this age group [1]. Currently, Streptococcus pneumoniae along with nontypeable Haemophilus influenzae are the major pathogens of AOM cases [2,3]. In a study of bacterial etiology of AOM between 2009 and 2010 in Bulgaria, the most frequently isolated pathogen was S. pneumoniae (47.6\%), followed by $H$. influenzae (27.6\%) [4].

Among the 93 pneumococcal serotypes, only a few are responsible for most cases of $\mathrm{AOM}$ in children worldwide, including serotypes 3, 6A, 6B, 9V, 14, 19A, 19F, and $23 \mathrm{~F}[5,6]$. Three pneumococcal conjugate vaccines (PCVs) are currently licensed for use in children and they have showed modest efficacy against AOM overall [7]. The 7-valent PCV was widely used throughout the world in the past 10 years. Recently, two new PCVs were introduced since 2010, PCV10 (Synflorix; GSK, UK) and PCV13 (Prevenar 13; Pfizer, USA). In children aged $<5$ years with AOM, the PCV7, PCV10 and PCV13 covered $54 \%, 60 \%$ and $85 \%$ of the pneumococcal serotypes, respectively in Germany [8].

Vaccination against pneumococcal diseases with PCV10 was introduced in Bulgaria in our national immunization program (NIP) for the first time in April, 2010. PCV7 was not used in Bulgaria prior to PCV10 introduction. Additionally, $H$. influenzae type $\mathrm{b}(\mathrm{Hib})$ conjugate vaccine was also included for routine immunization of infants as part of a pentavalent vaccine since 1 st April, 2010. Both conjugate vaccines are free of charge.

The prevalence of antibiotic-resistant S. pneumoniae has increased globally before the pre-vaccine era. The pattern of changes in antimicrobial susceptibility varies among serotypes and geographic regions [9]. Prior to PCV7 introduction, the major problem with antimicrobial resistance in $S$. pneumoniae worldwide was nonsusceptibility to penicillin, macrolides and multidrug resistance [10].

Resistance to $\beta$-lactams in $H$. influenzae, especially to ampicillin/amoxicillin, ranging from 3 to $65 \%$ in some countries is an increasing problem [11]. Although, ampicillin resistance is predominantly mediated by the $\beta$ lactamase production, alterations in penicillin-binding proteins (PBPs), mainly PBP 3, due to ftsI amino acid substitutions occur in some parts of the world [12]. In addition, $\beta$-lactamase positive, amoxicillin-clavulanate -resistant strains have been described due to the combination of both mechanisms [13].

The objectives of our study were to determine serotypes and antibiotic susceptibility of S. pneumoniae and $H$. influenzae isolates from middle ear fluid (MEF) cultures collected before the introduction of conjugate vaccines in the NIP. The presence of macrolide resistance determinants in S. pneumoniae isolates and the prevalence of mechanisms of ampicillin resistance in $H$. influenzae strains were also studied.

\section{Methods}

\section{Patients and isolates}

Pediartic (< 14 years of age) S. pneumoniae $(\mathrm{n}=128)$ and $H$. influenzae $(\mathrm{n}=40)$ isolates from middle ear fluid cultures obtained from children with the clinical diagnosis "acute otitis" complicated with otorrhea were examined. The AOM episodes were confirmed by an otorrinolaryngologist or pediatrician. The strains were isolated from several microbiological laboratories throughout Bulgaria between 1994 and 2011 and isolates had been sent in our department on a voluntary basis through a passive, laboratory-based study for retrospective analysis. Only one episode of AOM or one isolate of any of the two pathogens was included per patient, except for one child from whom both were detected from the same sample (mixed infection). Serotypes of $S$. pneumoniae were distributed per age group ( $\leq 2$ years, $2-5$ years, and $>5-14$ years); $90 \%$ of $H$. influenzae strains were isolated from children aged less than 5 years. None of the children with AOM had been immunized with pneumococcal or Hib conjugate vaccines. Five pneumococcal strains isolated in 2011 were also included because they were from children which had not received vaccines.

S. pneumoniae strains were confirmed with both methods - optochin susceptibility test and bile solubility. $H$. influenzae isolates were identified by Gram-stain morphology, growth requirements for factor $\mathrm{V}, \mathrm{X}$, and porphyrin (A.L.A. disks, Remel, Lenexa, Kansas, USA), biochemical tests according to standard methods and the presence of iga protease gene determined by conventional PCR $[14,15]$.

\section{Antimicrobial susceptibility test}

The antibiotic susceptibilities were determined by the broth microdilution method according to guidelines of Clinical and Laboratory Standards Institute (CLSI) [16]. Minimal inhibitory concentrations (MICs) were performed on Microtiter plates (Sensititre, Trek Diagnostic Systems Ltd., UK). STR6F MIC plate was inoculated with cationadjusted Mueller-Hinton broth and 5\% lysed horse blood for S. pneumoniae, and for H. influenzae HPB-Haemophilus/Streptococcus pneumoniae plate was inoculated with Haemophilus test broth. Final bacterial density was $5 \times$ $10^{5} \mathrm{CFU} / \mathrm{ml}$ and the plates were incubated at $35^{\circ} \mathrm{C}$ for 20-24 $\mathrm{h}$ in $5 \% \mathrm{CO}_{2}$. The MICs were interpreted according to CLSI, 2010 [16]. All H. influenzae strains were tested for $\beta$-lactamase production by the chromogenic cephalosporin test with nitrocefin as substrate. Standard quality control strains were S. pneumoniae ATCC 49619 and H. influenzae 
ATCC 49247. Strains resistant to $\geq 3$ classes of antibiotics were defined as multidrug resistant (MDR).

\section{Serotyping}

Serogrouping of S. pneumoniae was performed using the latex agglutination method (Pneumotest-Latex kit, Statens Serum Institut, Copenhagen, Denmark). Serotyping was performed later with the capsular Neufeld Quellung method by using some common factor antisera (SSI, Copenhagen, Denmark) [17]. H. influenzae strains were serotyped by a PCR capsule typing method, detecting the capsule-producing gene - bexA. The algorithm used for detection of the cap genes and for determining capsular serotypes was that proposed by Falla et al. [18].

\section{Detection of macrolide resistance determinants in $S$. pneumoniae}

The presence of $\operatorname{erm}(\mathrm{B})$ and $m e f(\mathrm{~A})$ was evaluated concurrently in a duplex reaction by PCR for all erythromycinresistant S. pneumoniae (ERSP) isolates. The primer sets were as described by Sutcliffe et al. [19]. Gene amplification was performed using a Techgene-thermal cycler (Techne, England). Mef(A) and $m e f(\mathrm{E})$ were then distinguished by PCR restriction fragment length polymorphism analysis of the 348-bp mef amplicon with BamHI restriction enzyme (New England Biolabs), which has no restriction site in $m e f(\mathrm{E})$ and one in $\operatorname{mef}(\mathrm{A})$, generating two fragments of 284 and $64 \mathrm{bp}$ [20]. Mutations in ribosomal protein L4 were determined as described elsewhere [21].

\section{DNA sequencing analysis of $\mathrm{ftsl}$ gene in $\mathrm{H}$. influenzae}

The DNA fragment encoding the transpeptidase region of PBP 3 was amplified by PCR from strains which were nonsusceptible to ampicillin (MICs of $\geq 2 \mu \mathrm{g} / \mathrm{ml}$ ). Amplification of ftsI gene was done using primers and PCR conditions described previously [22]. Nucleotide and amino acid sequences were analysed using DNAMAN 4.11 Software (Lynnon BioSoft, Canada). Deduced amino acid sequences were compared with the reference sequence obtained from the $H$. influenzae $\mathrm{Rd}$ strain. Strain ATCC 49247 was included as an H. influenzae BLNAR control strain.

We classified $H$. influenzae strains according to their ampicillin susceptibility, $\beta$-lactamase production, and on the basis of the DNA sequencing results obtained from ampicillin-nonsusceptible isolates. Four main classes were determined: $\beta$-lactamase negative, ampicillin-susceptible strains with $\mathrm{MIC}$ of $\leq 1 \mu \mathrm{g} / \mathrm{ml}$ (BLNAS); strains producing $\beta$-lactamase, and without mutations in the ftsI gene (BLPAR); $\beta$-lactamase negative, ampicillin-nonsusceptible strains (MICs of $\geq 2 \mu \mathrm{g} / \mathrm{ml}$ ) which have amino acid substitutions in the ftsI gene (BLNAR); and isolates that have both mechanisms ( $\beta$-lactamase production and amino acid substitutions in the ftsI gene)(BLPACR).

\section{Results}

Serotype distribution among S. pneumoniae and $H$. influenzae

Serotypes of $128 \mathrm{~S}$. pneumoniae isolates obtained from MEF are presented in Table 1 . The strains are divided in three age groups: children aged $<2$ years $(50.0 \%)$, children aged $>2$ to 5 years $(35.9 \%)$, and children aged $>5 \mathrm{y}-14 \mathrm{y}$

Table 1 Serotype distribution of S. pneumoniae isolates from children with acute otitis media by age group and potential vaccine coverige: 1994-2011

\begin{tabular}{|c|c|c|c|c|c|}
\hline \multirow[t]{2}{*}{ Serotype } & \multicolumn{4}{|c|}{ No. of isolates per age group } & \multirow{2}{*}{$\begin{array}{l}\text { Total no. } \\
(\%) \text { of } \\
\text { particular } \\
\text { serotype }\end{array}$} \\
\hline & $\leq 2 \mathrm{y}$ & $>2-5$ y & $\leq 5 \mathrm{y}$ & $>5 y-14 y$ & \\
\hline \multicolumn{6}{|c|}{ PCV7 serotypes } \\
\hline $19 \mathrm{~F}$ & 17 & 7 & 24 & 2 & $26(20.3)$ \\
\hline $6 \mathrm{~B}$ & 8 & 9 & 17 & 3 & $20(15.6)$ \\
\hline 14 & 10 & - & 10 & 1 & $11(8.6)$ \\
\hline $9 \mathrm{~V}$ & 5 & 3 & 8 & 3 & $11(8.6)$ \\
\hline $23 \mathrm{~F}$ & 5 & 4 & 9 & 1 & $10(7.8)$ \\
\hline $18 \mathrm{C}$ & 1 & 1 & 2 & 1 & $3(2.3)$ \\
\hline Total no. & 46 & 24 & 70 & 11 & $81(63.3)$ \\
\hline
\end{tabular}

Other vaccine serotypes

$\begin{array}{lccccc}19 \mathrm{~A} & 9 & 4 & \mathbf{1 3} & 1 & 14(10.9) \\ 3 & 2 & 3 & \mathbf{5} & 1 & 6(4.7) \\ 6 \mathrm{~A} & 1 & 2 & \mathbf{3} & 1 & 4(3.1) \\ 7 \mathrm{~F} & 1 & 1 & \mathbf{2} & - & 2(1.6) \\ 1 & - & 1 & \mathbf{1} & 1 & 2(1.6) \\ \text { Total no. } & 13 & 11 & \mathbf{2 4} & 4 & 28(21.9)\end{array}$

Non-vaccine serotypes

$\begin{array}{lccccc}15 \mathrm{~B} & 2 & 3 & \mathbf{5} & - & 5(3.9) \\ 23 \mathrm{~A} & 2 & 1 & \mathbf{3} & - & 3(2.3) \\ 11^{\mathrm{a}} & - & 2 & \mathbf{2} & - & 2(1.6) \\ 13,28^{\mathrm{b}} & - & 1 & \mathbf{1} & 1 & 2(1.6) \\ 15 \mathrm{C} & - & 2 & \mathbf{2} & - & 2(1.6) \\ 10^{\mathrm{a}} & 1 & 1 & \mathbf{2} & - & 2(1.6) \\ 16 / 36 / 37^{\mathrm{b}} & - & - & - & 1 & 1(0.8) \\ 24 / 31 / 40^{\mathrm{b}} & - & 1 & \mathbf{1} & - & 1(0.8) \\ \mathrm{N} T^{\mathrm{c}} & - & - & - & 1 & 1(0.8) \\ \text { Total no. } & 5 & 11 & \mathbf{1 6} & 3 & 19(14.8) \\ \text { Overall } & 64(50.0) & 46(35.9) & \mathbf{1 1 0 ( 8 5 . 9 )} & 18(14.1) & 128(100.0)\end{array}$

no. (\%)

Percentage coverage rate of pneumococcal conjugate vaccines

$\begin{array}{llllll}\text { PCV7 } & 71.9 & 52.2 & \mathbf{6 3 . 6} & 61.1 & 63.3 \\ \text { PCV10 } & 73.4 & 56.5 & \mathbf{6 6 . 4} & 66.7 & 66.4 \\ \text { PCV13 } & 92.2 & 76.1 & \mathbf{8 5 . 5} & 83.3 & 85.2\end{array}$

${ }^{a}$ The strains were determined to serogroup.

${ }^{b}$ Expected to be serotypes/serogroups (Strains were positive only for one of the pooled sera).

c Non-typeable strains with Pneumotest-kit sera. 
(14.1\%). The leading serotype among all children with pneumococcal AOM was $19 \mathrm{~F}$ (20.3\%), followed by serotypes 6B (15.6\%), 19A (10.9\%), serotypes 14 and $9 \mathrm{~V}(8.6 \%$ each), and $23 \mathrm{~F}$ (7.8\%). Serotypes 19A, 3 and 6A included in the PCV13 accounting altogether $18.7 \%$ among pneumococcal strains.

Coverage by serotypes included in the current PCV was evaluated and the calculations were performed without accounting for possible cross-protection. The potential coverage rates by the PCV7, PCV10 and PCV13 for children aged $<5$ years were $63.6 \%, 66.4 \%$ and $85.5 \%$, respectively.

Forty MEF isolates from children with AOM were identified as $H$. influenzae. Nontypeable $H$. influenzae (NTHi) strains predominated $(\mathrm{n}=39 ; 97.5 \%)$, and one was encapsulated serotype a isolate (2.5\%).

\section{Antimicrobial susceptibility}

Antibiotic susceptibility data for 128 S. pneumoniae isolates are presented in Table 2 . Reduced susceptibility to penicillin (oral penicillin V) was seen in $68.1 \%$ of the strains. This included strains $(28.1 \%)$ with high level of resistance to penicillin $(\mathrm{MIC} \geq 2 \mathrm{mg} / \mathrm{L})$. The rate of resistance to macrolides (erythromycin) was $46.9 \%$. Susceptibility results to amoxicillin shows $6.2 \%$ intermediately resistant strains and $4.7 \%$ fully resistant isolates. In a pre-vaccine era the percentage of multidrug-resistant strains was $53.9 \%$ $(69 / 128)$. In order to observe any changes in antibiotic resistance over the time, all pneumococcal strains were divided in two periods depending on the year when the strain was isolated: 1994-2005 and the more representative pre-vaccine period 2006-2011 (Table 2). During the second period we revealed increased resistance to erythromycin from $36.7 \%$ to $53.2 \%$, and to clindamycin from $22.4 \%$ to $40.5 \%$, respectively. Non-susceptibility rates to amoxicillin, cefuroxime sodium and ceftriaxone were found in $8.2 \%, 28.6 \%$ and $4.1 \%$, respectively in the first period, and were increased up to $12.7 \%, 41.8 \%$ and $8.9 \%$, respectively in the second period 2006-2011.

A total of $40 \mathrm{H}$. influenzae strains were tested for susceptibility to antibiotics. High-nonsusceptibility rates were found to trimethoprim-sulfamethoxazole - $35.0 \%$ (14/40), and to ampicillin - 25.0\% (10/40). Of the 10 ampicillin nonsusceptible $H$. influenzae strains, 9 were fully resistant to ampicillin ( $\mathrm{MIC} \geq 4 \mathrm{mg} / \mathrm{L}$ ) and one was intermediately susceptible $(\mathrm{MIC}=2 \mathrm{mg} / \mathrm{L})$ isolate (Table 3). Ceftriaxone and levofloxacin were the most active agents tested, and all isolates were fully susceptible to these agents.

\section{Macrolide resistance determinants among S. pneumoniae isolates}

Of the 128 MEF isolates 60 (46.9\%) were ERSP. The distribution of macrolide resistance determinants and particular serotypes are presented in Table 4 . We found erm (B) gene in 34 isolates $(56.7 \%)$, mef(E) gene in 15 isolates (25.0\%), 9 isolates harbored both genes $\operatorname{erm}(\mathrm{B})+\operatorname{mef}(\mathrm{E})$ (15.0\%), and 2 (3.3\%) strains had mutations of L4 ribosomal protein. The predominant serotypes among erm(B) genotype were $6 B(n=16), 19 A(n=9)$, and $19 F(n=6)$. Of the 15 isolates harboring mef(E) gene the prevalent serotypes were $14(\mathrm{n}=10)$ and $19 \mathrm{~F}(\mathrm{n}=3)$. The dual resistance mechanism $\operatorname{erm}(\mathrm{B})+\operatorname{mef}(\mathrm{E})$ was found in $19 \mathrm{~F}$ $(\mathrm{n}=7)$ and 19A $(\mathrm{n}=2)$ serotypes. Of note, resistance mechanism L4 found in two strains isolated in 1995 has never been seen in the years after. The coverage rates of the PCV7, PCV10 and PCV13 for ERSP were $75.0 \%$, $75.0 \%$ and $96.7 \%$, respectively. Considering co-resistance rates to penicillin according to macrolide resistance genotype, the percentage of strains exhibiting non-

Table 2 Antimicrobial susceptibility of 128 S. pneumoniae isolates from middle ear fluids and trends in antibiotic nonsusceptibility divided in two periods depending on year of strain isolation

\begin{tabular}{|c|c|c|c|c|c|c|c|}
\hline \multirow[t]{2}{*}{ Antibiotics } & \multicolumn{2}{|c|}{ MICs $(\mu \mathrm{g} / \mathrm{mL})$} & \multicolumn{3}{|c|}{ Overall\% susceptibility $n=128$} & \multirow{2}{*}{$\begin{array}{c}\text { NS rates in } 1994-2005 n=49 \\
\%\end{array}$} & \multirow{2}{*}{$\begin{array}{c}\text { NS rates in } 2006-2011 \mathrm{n}=79 \\
\%\end{array}$} \\
\hline & $\mathrm{MIC}_{50 / 90}$ & MIC range & $S$ & I & $\mathbf{R}$ & & \\
\hline Penicillin & $0.12 / 4.0$ & $0.01-8.0$ & 31.2 & 40.6 & 28.1 & 73.5 & 65.8 \\
\hline Amoxicillin & $0.12 / 4.0$ & $0.01-8.0$ & 89.1 & 6.2 & 4.7 & 8.2 & 12.7 \\
\hline Cefuroxime sodium & $\leq 0.5 / 8.0$ & $\leq 0.5-\geq 16.0$ & 63.3 & 5.5 & 31.2 & 28.6 & 41.8 \\
\hline Ceftriaxone & $0.06 / 1.0$ & $0.03-4.0$ & 93.0 & 4.7 & 2.3 & 4.1 & 8.9 \\
\hline Erythromycin & $\leq 0.25 / \geq 64.0$ & $\leq 0.25-\geq 64.0$ & 53.1 & 0.0 & 46.9 & 36.7 & 53.2 \\
\hline Clindamycin & $0.06 / \geq 64.0$ & $0.01-\geq 64.0$ & 66.4 & 0.8 & 32.8 & 22.4 & 40.5 \\
\hline Levofloxacin & $1.0 / 1.0$ & $0.5-2.0$ & 100.0 & 0.0 & 0.0 & 0.0 & 0.0 \\
\hline Tetracycline & $2.0 / \geq 8.0$ & $0.25-\geq 8.0$ & 50.8 & 0.8 & 48.4 & 46.9 & 50.6 \\
\hline Chloramphenicol & $4.0 / \geq 8.0$ & $0.5-\geq 8.0$ & 84.4 & - & 15.6 & 24.5 & 10.1 \\
\hline Trimeth/Sulfameth & $2.0 / \geq 4.0$ & $0.06-\geq 4.0$ & 35.2 & 22.7 & 42.2 & 65.3 & 64.6 \\
\hline
\end{tabular}

MICs-minimal inhibitory concentracions; $M I C_{50 / 90}$, MICs for $50 \%$ and $90 \%$ of the isolates, respectively; $R$-resistant; $I$-intermediate; $S$-susceptible (Interpretation according to CLSI, 2010); NS - nonsusceptibility; The following breakpoints ( $\mu \mathrm{g} / \mathrm{mL}$ ) for penicillin (oral penicillin V) were used: susceptible $\leq 0.06$; intermediate $\geq$ 0.12 - 1.0; resistant $\geq 2$; Trimethoprim/Sulfamethoxazole (1:19 ratio), MIC refers to trimethoprim value. 
Table 3 Distribution of ampicillin resistance classes of paediatric middle ear fluids $\boldsymbol{H}$. influenzae isolates according to their $\beta$-lactamase production, susceptibilities to ampicillin and amoxiclav, and amino acid substitutions present in ampicillin nonsusceptible strains with mutations in $\mathrm{ftsl}$ gene

\begin{tabular}{|c|c|c|c|c|c|c|}
\hline \multirow{2}{*}{$\begin{array}{l}\text { Resistance } \\
\text { class }\end{array}$} & \multirow[t]{2}{*}{ No. of strains (\%) } & \multirow[t]{2}{*}{$\beta$-lac } & \multicolumn{2}{|c|}{ MIC (mg/L) } & \multirow[t]{2}{*}{ Amino acid substitution(s) in ftsl gene } & \multirow[t]{2}{*}{ Group } \\
\hline & & & Ampicillin & Amoxiclav & & \\
\hline BLNAS & $30(75.0)$ & - & $\leq 1.0$ & $\leq 2.0$ & ND & - \\
\hline BLPAR & $4(10.0)$ & + & $\geq 8.0$ & $\leq 2.0$ & No changes & - \\
\hline BLNAR & $1(2.5)$ & - & 4.0 & 4.0 & Arg517His & । \\
\hline BLNAR & $1(2.5)$ & - & 2.0 & 4.0 & Asn526Lys & $\| a$ \\
\hline BLPACR & $1(2.5)$ & + & $\geq 8.0$ & 4.0 & Asp350Asn, Gly490Glu, Asn526Lys, Ala530Ser & $\| a$ \\
\hline BLNAR & $2(5.0)$ & - & 4.0 & 8.0 & Asp350Asn, Met377lle, Gly490Glu, Ala502Val, and Asn526Lys & $\| \mathrm{b}$ \\
\hline BLNAR & $1(2.5)$ & - & 4.0 & 8.0 & Asp350Asn, Ala502Thr, Asn526Lys & \|c \\
\hline Total & $40(100.0)$ & & & & & \\
\hline
\end{tabular}

Abbreviations: $\beta$-lac, $\beta$-lactamase production; Amoxiclav, amoxicillin-clavulanic acid (2:1 ratio). MIC refers to amoxicillin value. ND, DNA sequencing of ftsl gene was not done; BLNAS, $\beta$-lactamase negative, ampicillin-susceptible strains; BLPAR, $\beta$-lactamase positive ampicillin-resistant $H$. influenzae without amino acid substitutions in the PBP 3; BLNAR, $\beta$-lactamase negative ampicillin-resistant $H$. influenzae with amino acid substitutions in the PBP 3 ; BLPACR, $\beta$-lactamase positive strains with amino acid substitutions in the PBP 3; No changes along the section of amino acids 350 to 530 . The strains with ftsl gene mutations were classified into groups according to the proposed criteria by Dabernat et al. [22].

susceptibility to oral penicillin (MIC $\geq 0.1 \mathrm{mg} / \mathrm{L}$ ) was found in 93.3\% of ERSP isolates overall. The proportion of fully resistant to penicillin ERSP isolates was $17.6 \%$ in strains harboring erm(B) gene, $20.0 \%$ in these carried $m e f(E)$ gene, whereas $66.7 \%$ of isolates showing the dual resistance mechanism $\operatorname{erm}(\mathrm{B})+m e f(\mathrm{E})$ were fully resistant to oral penicillin.

\section{Ampicillin-resistant mechanisms and amino acid} substitutions of PBP 3 among MEF $H$. influenzae isolates The total collection of $40 \mathrm{H}$. influenzae was grouped according to our definition of ampicillin resistance classes: BLNAS strains $(\mathrm{n}=30 ; 75.0 \%)$, BLPAR $(\mathrm{n}=4 ; 10.0 \%)$ isolates without mutations in the ftsI gene, BLNAR strains $(\mathrm{n}=5 ; 12.5 \%)$ with amino acid substitutions in the ftsI gene, and BLPACR $(\mathrm{n}=1 ; 2.5 \%)$ strain that was $\beta$-lactamase positive, and had mutations in the critical region of ftsI gene with amino acid substitutions in PBP 3. Table 3 shows these ampicillin-resistance classes according to their $\beta$-lactamase production, susceptibilities to ampicillin and amoxicillin-clavulanic acid, and amino acid substitutions which present in ampicillin nonsusceptible strains. Of note, among six strains with amino acid substitutions in PBP 3 (5 BLNAR and one BLPACR), three were resistant to amoxicillin-clavulanic acid, and three strains were susceptible according to CLSI breakpoints.

The nucleotide sequences of the ftsI gene were determined for 10 ampicillin-nonsusceptible $H$. influenzae strains (MICs $\geq 2 \mu \mathrm{g} / \mathrm{ml}$ ) and their deduced amino acid sequences were compared with those of the ampicillinsusceptible $\mathrm{Rd}$ strain. Five $\beta$-lactamase nonproducing strains had significant amino acid substitutions in the ftsI gene, thereby we categorizing them as BLNAR strains. Among remaining $5 \beta$-lactamase positive strains, one isolate had amino acid substitutions in PBP 3. According to the classification of Dabernat et al. [22] different mutation patterns were identified among BLNAR and BLPACR strains. The Arg517His substitution that defines group I was observed in one ampicillin nonsusceptible $H$. influenzae. Most isolates $(\mathrm{n}=5)$ belonged to group II, defined by the Asn526Lys substitution, and

Table 4 Genotype distribution of 60 erythromycin-resistant S. pneumoniae (ERSP) isolates from middle ear fluids: association with serotypes and co-resistance to penicillin

\begin{tabular}{|c|c|c|c|c|c|}
\hline \multirow{2}{*}{$\begin{array}{l}\text { Resistance } \\
\text { genotype }\end{array}$} & \multirow{2}{*}{$\begin{array}{l}\text { Total no. }(\%) \\
\quad(n=128)\end{array}$} & \multicolumn{3}{|c|}{ Co-resistance to penicillin ${ }^{1} \mathrm{n}(\%)$} & \multirow[t]{2}{*}{ Serotypes of ERSP (n) } \\
\hline & & $\mathbf{R}$ & I & $\mathrm{S}$ & \\
\hline $\operatorname{erm}(\mathrm{B})$ & $34(56.7)$ & $6(17.6)$ & $25(73.5)$ & $3(8.8)$ & $6 \mathrm{~B}(16) ; 19 \mathrm{~A}(9) ; 19 \mathrm{~F}(6) ; 14,23 \mathrm{~F}, 23 \mathrm{~A}(1)$ each \\
\hline $\operatorname{mef}(\mathrm{E})$ & $15(25.0)$ & $3(20.0)$ & $11(73.3)$ & $1(6.7)$ & 14 (10); $19 \mathrm{~F}(3) ; 6 \mathrm{~B}, 15 \mathrm{~B}(1)$ each \\
\hline$e r m(B)+m e f(E)$ & $9(15.0)$ & $6(66.7)$ & $3(33.3)$ & - & $19 \mathrm{~F}(7) ; 19 \mathrm{~A}(2)$ \\
\hline $\mathrm{L}_{4}^{2}$ & $2(3.3)$ & $2(100.0)$ & - & - & $19 A(2)$ \\
\hline Total no. (\%) of ERSP & $60(46.9)$ & $17(28.3)$ & $39(65.0)$ & $4(6.7)$ & - \\
\hline
\end{tabular}

$R$-resistant; l-intermediate; S-susceptible (Interpretation according to CLSI, 2010); ${ }^{1}$ The following breakpoints ( $\mu \mathrm{g} / \mathrm{mL}$ ) for penicillin (oral penicillin V) were used: susceptible $\leq 0.06$; intermediate $\geq 0.12-1.0$; resistant $\geq 2 .{ }^{2}$ Mutations in ribosomal protein $L 4$ were determined as described elsewhere [21]. 
could be further subdivided into three subgroups - IIa, IIb and IIc. Group III mutations in ftsI gene conferring highlevel resistance were not detected.

\section{Discussion}

This is a retrospective study about serotype distribution and antibiotic resistance of MEF S. pneumoniae and $H$. influenzae isolates collected from children before introduction of PCV10 and Hib conjugate vaccines nationwide. Microbiological diagnosis of AOM is not done on a routine basis in Bulgaria and this mild disease is underrepresented in our data set. Also, the tympanocentesis is not a common practice in our country. Thus, bacterial cultures studied here most likely represent cases of severe otitus media or patients with a complicated AOM.

The analysis included $128 \mathrm{~S}$. pneumoniae isolates, of which $86 \%$ occurring in children aged $\leq 5$ years. We found a relatively high rate of serotype $19 \mathrm{~F}$ infections (20.3\%), which is in accordance with the serotype distribution observed in the studies of AOM in German children [8], and the Finland [7]. Serotypes $6 B$ and 19A were next most prominent, each comprising about $16 \%$ and $11 \%$, respectively of the overall data set. The coverage calculations for the serotypes included in PCV7 without cross-protection was $63.6 \%$ in children with AOM aged $<5$ years, which is almost the same as was the serotype coverage previously reported for invasive pneumococcal disease (IPD) for the same age group in Bulgaria [23]. The PCV10 increase the coverage by $2.8 \%$ of pneumococcal AOM (66.4\%). The PCV13, which includes three serotypes in addition to the PCV10, would increase the coverage to $85.5 \%$, mainly due to serotype 19A followed by serotypes 3 and 6A (Table 1). However the cross-protection of serotype $6 \mathrm{~B}$ towards serotype 6A was not included in calculations for our study. We found 14 serotype 19A isolates, 13 of which were multidrug resistant.

The results showed a high rate of reduced oral penicillin nonsusceptibility (68\%) and a very high rate of ERSP isolates (47\%) among MEF strains. These rates are more than twice as high as previously recorded with IPD isolates in Bulgaria studied during the same year period [23]. These high resistance rates may be due to clonal spread of certain MDR pneumococcal serotypes such as $6 \mathrm{~B}, 19 \mathrm{~F}$ and 19A. This resistance reflects the complexity of many of the severe otitis media episodes which included therapy failures. Notably, we observed that the percentages of macrolide, clindamycin, amoxicillin, cefuroxime sodium and ceftriaxone nonsusceptibility increased among $S$. pneumoniae strains isolated during the last 6 years of the study period. The high antibiotic consumption for the treatment of $\mathrm{AOM}$ represents the major pressure for resistance selection [24].
Overall, macrolide resistance in S. pneumoniae now is mainly due to $\mathrm{erm}$ (B) gene ( $71.7 \%$ ), including $15 \%$ of isolates with a dual macrolide resistance mechanism (Table 4). This pattern of macrolide resistance determinants in the last years replaced the efflux mechanism of macrolide resistance (mef genotype), which was predominant among ERSP in Bulgaria before 2005 [25]. Macrolide-resistant S. pneumoniae strains were found to be co-resistant to oral penicillin (Table 4), and to other compounds, such as tetracycline and trimethoprimsulfamethoxazole (data not shown). Thus, the prevalence of $M_{L} S_{B}$ genotype may be due to clonal spread of certain MDR pneumococcal serotypes as $6 \mathrm{~B}, 19 \mathrm{~F}$ and 19A. Interestingly, we observed multi-resistant serotype $19 \mathrm{~A}$ in many years ago. For the first time, this MDR serotype was found in 1995 with L4 mutations as the mechanism of macrolide resistance [21]. Since 2000 and after, erm (B) genotype was found in serotype 19A isolates (nine strains). Dual macrolide resistance mechanisms were observed recently, between 2008 and 2010 in two serotype 19A strains (Table 1 and Table 4). Both the emergence of a new clone or capsular switching might be associated with these changes in our 19A serotype isolates. A considerable increase in the disease caused by serotype 19A has been recorded after the introduction of PCV7 in some countries [24,26]. Of note, MDR serotype 19A isolates have also been identified in Israel and Finland before the large-scale use of conjugate vaccines as was the case in our study $[27,28]$.

Our findings of $97.5 \%$ nontypeable $H$. influenzae strains isolated from AOM are in accordance with the literature [11,29]. Nontypeable $H$. influenzae are the major etiologic agents of respiratory tract infections in our country as was shown before [30]. Ampicillin/ amoxicillin nonsusceptible $H$. influenzae were found to be a quarter of our MEF isolates. Among BLNAR and BLPACR most of the isolates (5/6) belonged to Dabernat group II, defined by the Asn526Lys substitution. A high incidence of BLNAR $H$. influenzae strains has been reported in Japan, Spain and France [11,12,22]. The emergence of BLNAR and BLPACR strains among MEF $H$. influenzae isolates may have implications for the treatment of AOM infections, because amoxicillin and amoxicillin-clavulanate are the most common antibiotics used in the treatment of AOM in our country.

The main limitations of our study are: 1) it covers a long period of time, and the power is low; 2) isolates were sent by microbiology labs in hospitals, and we do not have clinical information on the type of AOM; 3 ) as isolates came from hospitals, they are not representative of S. pneumoniae and H. influenzae isolates causing otitis in children attending primary medical centers; 4) the numbers of S. pneumoniae and $H$. influenzae strains are small, because only a portion of children with AOM in 
Bulgaria are tested. Despite these limitations, we believe that they may represent a rough estimate of the serotype distribution and antibiotic resistance among a group of children with complicated AOM. This information will be invaluable when planning vaccination strategies to protect against otitis media.

In conclusion, the levels of antibiotic resistance among $S$. pneumoniae and $H$. influenzae causing severe otitis media in children are high in our settings. This has important therapeutic implications because such antibioticresistant isolates compromise the choice of macrolides and amoxicillin as treatment of AOM episodes in children. The existence of MDR S. pneumoniae serotype $19 \mathrm{~A}$ before the active pneumococcal immunization is of particular concern. The rate of 15\% BLNAR and BLPACR strains among $H$. influenzae MEF isolates may have implications for the treatment of AOM. Ongoing surveillance is needed for both pathogens in order to observe any changes in serotypes prevalence and antibiotic resistance after introduction of conjugate vaccines in our country.

\section{Consent}

Written informed consent was obtained from the patient's guardian/parent/next in keen for publication of this report and any accompanying images

\section{Competing interests}

All authors declare that they have no competing interests.

\section{Authors' contributions}

LS designed the study, participated in the laboratory analyses and experiments, and drafted the manuscript. TK and AA carried out technical help and participated in the experiments. IM monitored the progress and supervised the manuscript. DN and TK assisted in clinical isolates gathering. All authors read and approved the final manuscript.

\section{Acknowledgments}

The study was partially supported by funds from Medical University of Sofia (Grant No. D35/2012). We thank all microbiological laboratories in Bulgaria for their cooperation and for providing the isolates included in this study.

\section{Author details}

${ }^{1}$ Department of Medical Microbiology, Medical University of Sofia, Faculty of Medicine, Sofia 1431, BULGARIA. ${ }^{2}$ Department of Microbiology, National Center of Infectious and Parasitic Diseases, Sofia 1504, BULGARIA.

Received: 8 January 2013 Accepted: 16 March 2013

Published: 25 March 2013

\section{References}

1. American Academy of Pediatrics Subcommittee on Management of Acute Otitis Media: Diagnosis and management of acute otitis media. Pediatrics 2004, 113:1451-1465.

2. Dupont D, Mahjoub-Messai F, François M, Doit C, Mariani-Kurkdjian P, Bidet $P$, et al: Evolving microbiology of complicated acute otitis media before and after introduction of the pneumococcal conjugate vaccine in France. Diagn Microbiol Infect Dis 2010, 68:89-92.

3. Palmu AAI, Herva E, Savolainen H, Karma P, Makela PH, Kilpi TM: Association of clinical signs and symptoms with bacterial findings in acute otitis media. Clin Infect Dis 2004, 38:234-242.

4. Nashev D, Velinov T, Kuzmanov A, Setchanova L, Perov N, Bizeva L, et al: Study of bacterial etiology of acute otitis media in Bulgaria before introduction of pneumococcal conjugate vaccine. Probl Inf Parasit Dis 2010, 38(2):9-11.

5. Block SL, Hedrick J, Harrison CJ, Tyler R, Smith A, Findlay R, Keegan E: Pneumococcal serotypes from acute otitis media in rural Kentucky. Pediatr Infect Dis J 2002, 21:859-865.

6. Rodgers GL, Arguedas A, Cohen R, Dagan R: Global serotype distribution among Streptococcus pneumoniae isolates causing otitis media in children:potential implications for pneumococcal conjugate vaccines. Vaccine 2009, 27:3802-3810.

7. Eskola J, Kilpi T, Palmu A, Jokinen J, Haapakoski J, Herva E, et al: Efficacy of a pneumococcal conjugate vaccine against acute otitis media. $N$ Eng/ J Med 2001, 344:403-409.

8. Van der Linden M, Reinert RR: Serotype distribution in pneumococcal acute otitis media with ruptured tympanic membrane or sepsis in Germany. Eur J Clin Microbiol Infect Dis 2010, 29:749-754.

9. Imöhl M, Reinert RR, van der Linden M: Regional differences in serotype distribution, pneumococcal vaccine coverage, and antimicrobial resistance of invasive pneumococcal disease among German federal states. Int J Med Microbiol 2010, 300:237-247.

10. Appelbaum PC: Resistance among Streptococcus pneumoniae: implications for drug selection. Clin Infect Dis 2002, 34:1613-1620.

11. Tristram S, Jacobs MR, Appelbaum PC: Antimicrobial resistance in Haemophilus influenzae. Clin Microbiol Rev 2007, 20:368-389.

12. Kubota T, Higa F, Kusano N, Nakasone I, Haranage S, Tateyama M, et al: Genetic analyses of beta-lactamase negative ampicillin-resistant strains of Haemophilus influenzae isolated in Okinawa, Japan. Jpn J Infect Dis 2006, 59:36-41.

13. Matic V, Bozdogan B, Jabobs MR, Ubukata K, Appelbaum PC: Contribution of beta-lactamase and PBP amino acid substitutions to amoxicillin/ clavulanate resistance in beta-lactamase-positive, amoxicillin/ clavulanate-resistant Haemophilus influenzae. J Antimicrob Chemother 2003, 52:1018-1021.

14. Ledeboer NA, Doern GV: Haemophilus. In Manual of clinical microbiology. 10th edition. Edited by Versalovic J, Carroll KC, Jorgensen JH, Funke G, Landry ML, Warnock DW. Washington, DC, USA: ASM Press; 2011:588-602.

15. Mukundan D, Esevit Z, Patel M, Marris CF, Gilsdorf JR: Pharyangeal colonization dynamics of Haemophilus influenzae and Haemophilus haemolyticus in healthy adult carriers. J Clin Microbiol 2007, 45:3207-3217.

16. Clinical and Laboratory Standards Institute: Performance standards for antimicrobial susceptibility testing, Approved standard M100-S20. 20th edition. Wayne, PA: CLSl; 2010.

17. Sorensen UBS: Typing of pneumococci by using 12 pooled antisera. J Clin Microbiol 1993, 31:2097-2100.

18. Falla TJ, Crook DW, Brophy LN, Maskell D, Kroll JS, Moxon ER: PCR for capsular typing of Haemophilus influenzae. J Clin Microbiol 1994, 32:2382-2386.

19. Sutcliffe J, Grebe T, Tait-Kamradt A, Wondrack L: Detection of erythromycin-resistant determinants by PCR. Antimicrob Agents Chemother 1996, 40:2562-2566.

20. Montanari MP, Mingoia M, Giovanetti E, Varaldo PE: Phenotypes and genotypes of erythromycin-resistant pneumococci in Italy. J Clin Microbiol 2003, 41:428-431.

21. Tait-Kamradt A, Davies T, Appelbaum PC, Depardieu F, Courvalin P, Petitpas J, et al: Two new mechanisms of macrolide resistance in clinical strains of Streptococcus pneumoniae from Eastern Europe and North America. Antimicrob Agents Chemother 2000, 44:3395-3401.

22. Dabernat H, Delmas C, Seguy M, Pelissier R, Faucon G, Bennamani S, et al: Diversity of beta-lactam resistance-conferring amino acid substitutions in penicillin-binding protein 3 of Haemophilus influenzae. Antimicrob Agents Chemother 2002, 46:2208-2218.

23. Setchanova L, Alexandrova A, Mitov I, Nashev D, Kantardjiev T, The Group for Microbiological surveillance of pneumococci: Serotype distribution and antimicrobial resistance of invasive streptococcus pneumoniae isolates in Bulgaria before the introduction of pneumococcal conjugate vaccine. J Chemother 2012, 24(1):12-17.

24. Fenoll A, Aguilar L, Cicoso M-D, Gimenez M-J, Robledo O, Granizo J-J: Increase in serotype 19A prevalence and amoxicillin non-susceptibility among paediatric Streptococcus pneumoniae isolates from middle ear fluid in a passive laboratory-based surveillance in Spain, 1997-2009. BMC Infect Dis 2011, 11:239.

25. Setchanova L, Ouzounova-Raykova V, Zhelezova G, Mitov I: Prevalence and macrolide resistance phenotypes and genotypes among clinical isolates 
of Streptococcus pneumoniae collected in Sofia, Bulgaria from 2001 to 2005. J Chemother 2007, 19(3):256-262.

26. Pichichero ME, Casey JR: Emergence of a multiresistant serotype 19A pneumococcal strain not included in the 7 -valent conjugate vaccine as an otopathogen in children. Jama 2007, 298:1772-1778.

27. Dagan R, Givon-Lavi N, Leibovitz E, Greenberg D, Porat N: Introduction and proliferation of multidrug-resistant Streptococcus pneumoniae serotype $19 \mathrm{~A}$ clones that cause acute otitis media in an unvaccinated population. J Infect Dis 2009, 199(6):776-785.

28. Siira L, Jalava J, Tissari P, Vaara M, Kaijalainen T, Virolainen A: Clonality behaind the increase of multidrug-resistance among non-invasive pneumococci in Southern Finland. Eur J Clin Microbiol Infect Dis 2012, 31:867-871.

29. Thanavala Y, Lugade AA: Role of nontypeable Haemophilus influenzae in otitis media and chronic obstructive pulmonary disease. Adv Otorhinolaryngol 2011, 72:170-175.

30. Setchanova L, Kostyanev T, Markovska R, Miloshev G, Mitov I: Serotypes, antimicrobial susceptibility and $\beta$-lactam resistance mechanisms of clinical Haemophilus influenzae isolates from Bulgaria in a prevaccination period. Scand J Inf Dis 2013, 45(2):81-87.

doi:10.1186/1476-0711-12-6

Cite this article as: Setchanova et al:: Microbiological characterization of Streptococcus pneumoniae and non-typeable Haemophilus influenzae isolates as primary causes of acute otitis media in Bulgarian children before the introduction of conjugate vaccines. Annals of Clinical Microbiology and Antimicrobials 2013 12:6.

\section{Submit your next manuscript to BioMed Central and take full advantage of:}

- Convenient online submission

- Thorough peer review

- No space constraints or color figure charges

- Immediate publication on acceptance

- Inclusion in PubMed, CAS, Scopus and Google Scholar

- Research which is freely available for redistribution 